\title{
Séparation de particules microniques par focalisation hydrodynamique
}

\author{
Dorra Salhi, Mauricio Hoyos et Pascal Kurowski ${ }^{\mathrm{a}}$ \\ Laboratoire de Physique et Mécanique des milieux Hétérogènes (UMR 7636), 10 rue Vauquelin, 75231 Paris Cedex 05, France
}

Reçu le 6 octobre 2003, accepté le 8 janvier 2004

\begin{abstract}
Résumé - La séparation de particules de taille micronique par la technique dite de SPLITT a mis en évidence des effets de migrations transverses non spécifiques, qui limitent l'efficacité de cette séparation dans le cas d'un mélange de plusieurs espèces. Des expériences en micropesanteur ont montré le rôle important joué par la diffusion hydrodynamique induite par cisaillement dans cette migration anormale. Une nouvelle cellule a donc été mise au point : les particules sont injectées au centre du canal dans l'épaisseur de la cellule et peuvent être focalisées dans n'importe quelle position dans l'épaisseur. La focalisation hydrodynamique ainsi contrôlée permet d'éloigner les particules des parois, où le cisaillement est maximal, afin de minimiser les effets des forces de portance et de diffusion. Les premiers tests de séparation binaire réalisés avec cette cellule de focalisation sont très encourageants puisqu'ils montrent une augmentation notable de l'efficacité, en termes de pureté des échantillons, comparée à celle obtenue avec la cellule de SPLITT.
\end{abstract}

Mots clés : Séparation binaire / focalisation hydrodynamique / diffusion induite par cisaillement / micropesanteur

\begin{abstract}
Micronic particles separation by hydrodynamic focusing method. Sorting of micron size particles by the SPLITT fractionation technique shows a non specific transversal migration, which is detrimental to separation efficiency of mixtures of different species. Experiments in microgravity showed that the shear-induced hydrodynamic diffusion plays an important role in this unexpected migration. Thus, a new cell has been built: particles are injected at the centre part of the channel and can be focused anywhere in the channel thickness. This controlled hydrodynamic focusing allows taking particles away from the walls where the shear rate is maximum, in order to minimize lift force and shear diffusion effects. The first binary separation tests using this hydrodynamic focusing device are very promising since they show an appreciable improvement on separation efficiency compared to that obtained by using a usual SPLITT channel.
\end{abstract}

Key words: Binary separation / hydrodynamic focusing / shear induced diffusion / microgravity

\section{Introduction}

La technique de SPLITT (Split-Flow Thin cell fractionation) est une technique de séparation binaire capable de séparer rapidement et en continu les espèces d'un mélange comme des macromolécules, des particules ou encore des cellules biologiques. Les espèces à séparer sont entraînées par le liquide vecteur, généralement de l'eau, le long d'un canal rectangulaire de type Hele-Shaw, comprenant deux entrées $\left(a^{\prime}\right.$ et $\left.b^{\prime}\right)$, deux sorties (a et b) et un séparateur de flux à chaque extrémité (Fig. 1). Au cours de l'entraînement, ces espèces injectées subissent une migration transverse (dans l'épaisseur de la cellule)

\footnotetext{
${ }^{a}$ Auteur correspondant : pascal@pmmh.espci.fr
}

due généralement à l'effet de champs de forces transverses (champ gravitationnel, électrique, magnétique). Elles doivent alors traverser une zone de transport de faible épaisseur (analogue à une membrane virtuelle) pour sortir en b. Cette zone de transport est délimitée par deux plans de séparation :

- Un plan de séparation à l'entrée, ISP (« Inlet Splitting Plane $»)$ dont la position $W_{\mathrm{a}^{\prime}}$ dépend du débit d'injection $Q_{\mathrm{a}^{\prime}}$ et divise l'échantillon entrant en a' du liquide vecteur entrant en $\mathrm{b}^{\prime}$.

- Un plan de séparation à la sortie, OSP (« Outlet Splitting Plane ») qui sépare la couche de fluide qui sortira en a de celle qui sortira en b et dont la position $W_{\mathrm{a}}$ dépend par conséquent du débit de sortie $Q_{\mathrm{a}}$. 


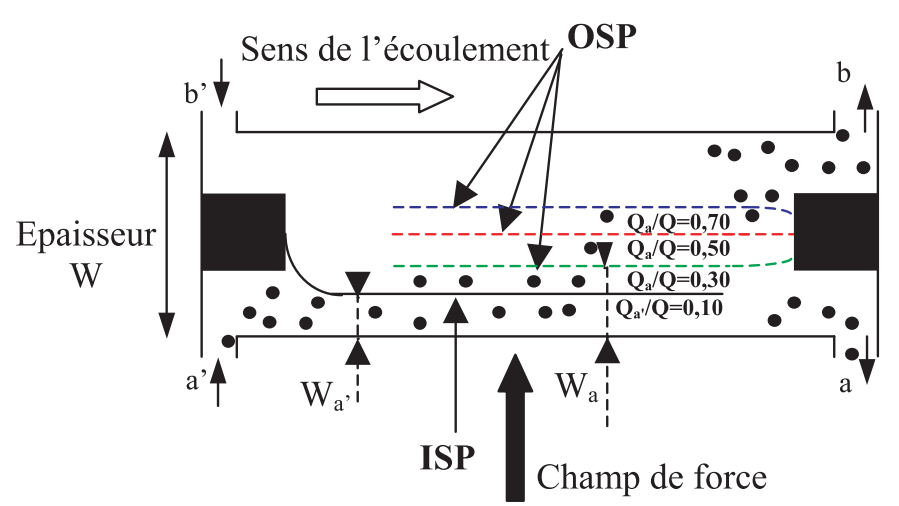

Fig. 1. Schéma d'une cellule de SPLITT et illustration de la zone de transport pour une position donnée de l'ISP : $Q_{\mathrm{a}^{\prime}} / Q=$ 0,10 et trois positions différentes de l'OSP : $Q_{\mathrm{a}} / Q=0,30$; 0,$50 ; 0,70 ; Q$ est le débit total.

Pour plus de détails sur la technique de SPLITT, on pourra se reporter à l'article de Giddings [1]. La séparation est quantifiée par la détermination du rapport entre la masse de particules $m_{\mathrm{a}}$ ou $m_{\mathrm{b}}$ éluées par la sortie a ou b, et la masse totale de particules injectées $m$ : $F_{\mathrm{a}}=m_{\mathrm{a}} / m$ ou $F_{\mathrm{b}}=m_{\mathrm{b}} / m$. La méthode de SPLITT est adaptée aux séparations binaires car deux fractions distinctes peuvent être collectées simultanément.

Le champ gravitationnel est le champ utilisé le plus fréquemment, notamment en vue d'applications liées à l'environnement. Mais des expériences récentes [2] visant à trier des particules magnétiques, des cellules souches du sang font appel à un champ magnétique. L'objectif est d'extraire des cellules souches du sang, d'échantillons contenant tous ses composants, à savoir, des globules rouges, des globules blancs, des plaquettes, etc. La rareté des cellules souches nécessite d'en récupérer le plus possible et donc d'utiliser une zone de transport étroite. Mais l'utilisation d'une zone de transport étroite a mis en évidence l'existence d'une migration transverse anormale ou non spécifique des particules, c'est-à-dire que des espèces non affectées par le champ, migrent malgré tout transversalement et traversent la zone de transport.

\section{2 Étude de la migration transverse non spécifique}

La migration transverse non spécifique observée expérimentalement dans toutes les cellules de SPLITT nuit dans la plupart des cas aux séparations. Il faut donc pour l'étudier supprimer tout champ de force extérieur, en particulier la gravité. Nous avons donc utilisé une cellule de SPLITT placée en position verticale, l'écoulement étant dans le même sens que la gravité (Fig. 2). Dans le cas idéal, les particules injectées en a' devraient sortir par la sortie opposée a : la fraction massique à la sortie b, $F_{\mathrm{b}}$, devrait toujours être nulle, quelle que soit l'épaisseur $\delta W=\left(W_{\mathrm{a}}-W_{\mathrm{a}^{\prime}}\right)$ de la zone de transport. Une étude faite par Hoyos et al. [3] met en évidence et quantifie cet effet de migration transverse : à $\delta W$ faible, $F_{\mathrm{b}}$ n'est jamais nulle
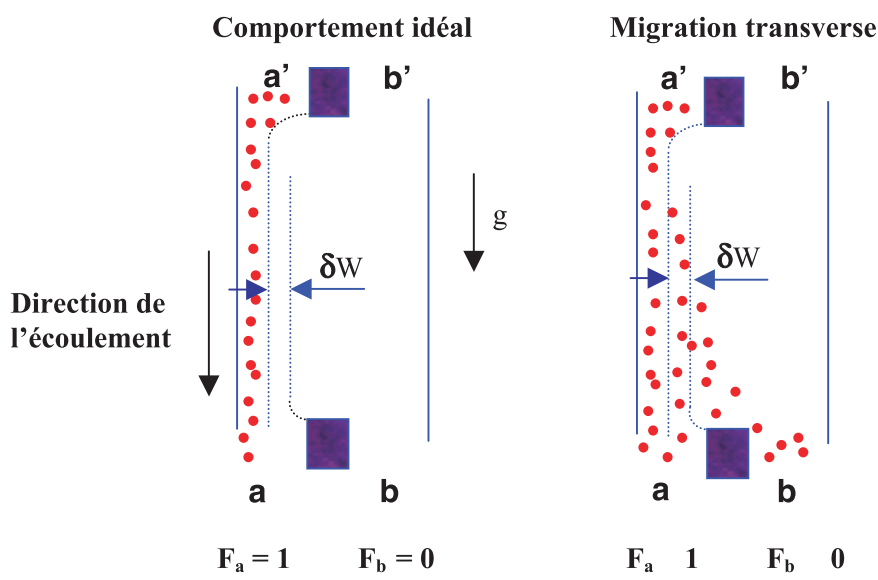

Fig. 2. Cellule de SPLITT placée verticalement : même en l'absence de champ de force appliqué, une migration transverse des espèces injectées est observée.

et décroît quand $\delta W$ augmente. Nous avons montré que les deux causes principales de cette migration transverse sont les forces de portance hydrodynamique et la diffusion induite par cisaillement. D'autres effets peuvent intervenir comme les imperfections du canal mais des études récentes [4] ont montré que cet effet est négligeable.

\subsection{Forces de portance hydrodynamique}

Des particules entraînées par un liquide au voisinage d'une paroi subissent une force perpendiculaire à leur vitesse, appelée portance ou force de lift. Cette force d'origine très différente de celle plus classique liée à l'effet Magnus, se manifeste même à des nombres de Reynolds relativement faibles. Son origine physique a motivé un grand nombre de travaux, tant théoriques $[5,6]$, qu'expérimentaux $[7,8]$. L'effet des forces de portance est d'éloigner de la paroi une particule qui se déplace à son voisinage.

En utilisant la théorie de Vasseur et Cox [6], si l'injection des particules dans une cellule de SPLITT se fait près d'une paroi (entrée a') de haut en bas, les particules auront tendance à se concentrer vers la paroi, et dans le cas contraire, les forces vont pousser les particules vers le centre du canal et par conséquent un plus grand nombre de particules sortiront en b (Fig. 3). Cet effet a bien été mis en évidence par Hoyos et Kurowski [9]. Mais la valeur de $F_{\mathrm{b}}$ est malgré tout bien trop grande pour pouvoir être expliquée à partir des seules forces de portance hydrodynamique. Cette section souligne le fait que la diffusion hydrodynamique induite par cisaillement joue un rôle, qui plus est majeur, dans la migration non spécifique des espèces.

\subsection{Diffusion induite par cisaillement}

La diffusion hydrodynamique induite par cisaillement est un phénomène de type collectif d'interaction entre particules qui migrent dans un canal à un champ de vitesse 


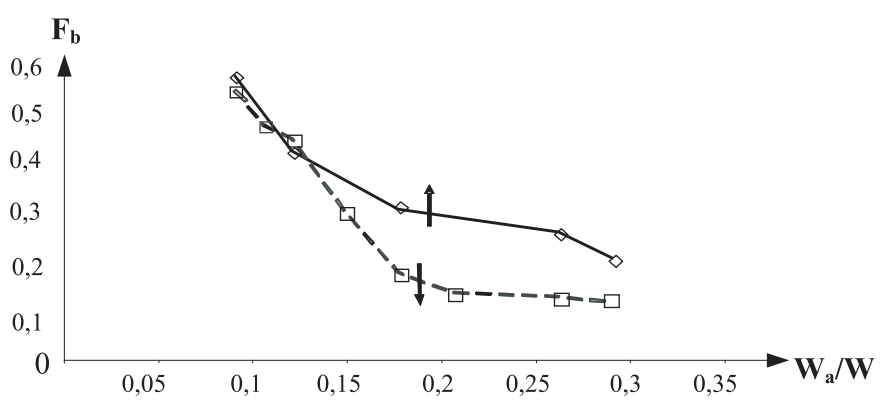

Fig. 3. Fraction massique $F_{\mathrm{b}}$ en fonction de l'épaisseur $W_{\mathrm{a}} / W$ de la zone de transport : la courbe en trait continu correspond à un écoulement de bas en haut (opposé à la gravité) tandis que la courbe en pointillés à un écoulement de haut en bas (même sens que la gravité).

non uniforme. Ainsi, des particules qui sont entraînées sur des lignes de courant plus rapides, dépassent des particules en écoulement sur des lignes plus lentes. L'interaction hydrodynamique entre particules produit une perturbation qui les fait sauter d'une ligne de courant à une autre. Le résultat net est un déplacement moyen des particules vers le centre du canal, perpendiculaire à la direction de l'écoulement. Cette diffusion hydrodynamique se caractérise par un coefficient de diffusion effectif $D_{\text {eff }}$ qui est proportionnel au taux de cisaillement $\dot{\gamma}$, au diamètre des particules $d$ au carré et à une fonction de la concentration $\phi$ (fraction volumique). Leighton et Acrivos [10] ont établi une expression semi-empirique de cette fonction qui est quadratique en $\phi$ pour de faible concentration. La limite entre les faibles et les fortes concentrations n'est pas très bien définie mais est de l'ordre de $10 \%$, donc dans tous les cas très supérieure à la concentration utilisée dans nos expériences (environ 0,5\%). Signalons que dans notre cas, le coefficient de diffusion brownienne $D$ est de l'ordre de $9 \times 10^{-10} \mathrm{~cm}^{2} / \mathrm{s}$ pour des particules de $5 \mu \mathrm{m}$ de diamètre, ce qui correspond à un nombre de Péclet $P_{e}=\dot{\gamma} a^{2} / D$ de $2,8 \times 10^{4}$ lorsque $\dot{\gamma}=100 \mathrm{~s}^{-1}$. Dans ces conditions, nos particules (de diamètre $5 \mu \mathrm{m}$ et $8 \mu \mathrm{m}$ ) peuvent être considérées comme non browniennes. Les valeurs de coefficient de diffusion induit par cisaillement déduites de nos courbes expérimentales sont quant à elles de l'ordre de $10^{-6} \mathrm{~cm}^{2} / \mathrm{s}$ [3] donc trois ordres de grandeur plus grand que le coefficient de diffusion brownienne.

La diffusion induite par cisaillement a suscité de nombreuses études théoriques et expérimentales [11-13]. Le modèle le plus proche de notre configuration expérimentale correspond à celui de la re-suspension visqueuse proposé par Leighton et Acrivos [10]. En effet, si la cellule est placée horizontalement, l'injection s'effectuant par l'entrée a' dans la partie inférieure de la cellule, la suspension sera soumise d'une part à la diffusion hydrodynamique qui l'éloigne de la paroi, et d'autre part à la pesanteur qui tend à la faire sédimenter.

Si l'épaisseur de la zone de transport est grande, l'efficacité de la séparation sera réduite mais les effets néfastes de la diffusion transverse seront réduits. L'épaisseur

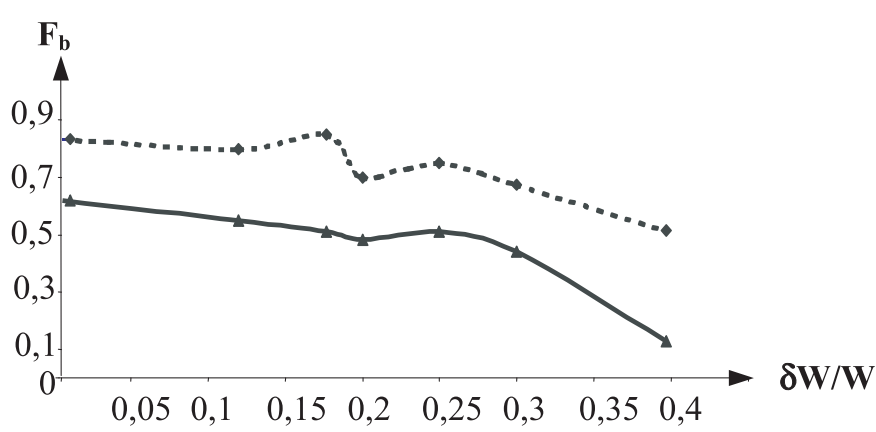

Fig. 4. Fraction volumique $F_{\mathrm{b}}$ en fonction de $\delta W / W$. Courbe en trait continu : expériences réalisées au sol $\left(g / g_{\mathrm{ref}}=1\right)$, courbe en pointillés : expériences réalisées en micropesanteur $\left(g / g_{\text {ref }}=0,02\right)$.

optimale $\delta W_{\text {opt }}$ de la zone de transport est alors définie par une longueur de diffusion effective construite à partir du coefficient de diffusion effectif $: l_{\text {eff }} \sim\left\{D_{\text {eff }} V / Q\right\}^{1 / 2}=$ $\delta W_{\text {opt }}$ où $V$ est le volume de la cellule.

\subsection{Expériences en micropesanteur}

Afin de découpler et de quantifier les effets de resuspension (dus à la gravité) des effets de diffusion hydrodynamique induite par cisaillement (indépendants de $g$ ), il est nécessaire de comparer les expériences réalisées au sol et les expériences réalisées en micropesanteur. Cellesci ont été faites lors de la campagne de vols paraboliques de mars 2002 à Bordeaux, organisée par le CNES, dans un Airbus A300 spécialement aménagé pour ce type de vols. L'avion effectue au total 90 paraboles (30 par jour) pendant lesquelles l'expérimentateur et l'expérience se retrouvent en micropesanteur $\left(g / g_{\text {ref }} \sim 0,02\right)$ pendant 20 secondes environ. Des particules de silice de $5 \mu \mathrm{m}$ sont injectées en a'. Le liquide vecteur utilisé est de l'eau et la cellule de SPLITT est placée horizontalement lors des expériences au sol. Nous avons tracé sur la figure 4 la fraction de particules récupérées à la sortie $\mathrm{b}, F_{\mathrm{b}}$, en fonction de $Q_{\mathrm{a}} / Q$ donc de l'épaisseur $\delta W$ de la zone de transport (voir Fig. 1). Sur la même figure, nous avons porté les résultats d'expériences faites au sol $\left(g / g_{\text {ref }}=1\right)$ ainsi que les résultats obtenus en micropesanteur.

On remarque qu'à $g / g_{\text {ref }}=1, F_{\mathrm{b}}$ n'est jamais nul, sauf pour des zones de transport très larges $(\delta W / W>0,4)$ ce qui montre que la sédimentation ne compense jamais complètement l'effet diffusif induit par le cisaillement. À $\delta W / W$ donné, la valeur de $F_{\mathrm{b}}$ est plus importante en micropesanteur qu'au sol car nous avons éliminé la sédimentation. L'écart entre les deux courbes semble constant ce qui veut dire que les deux effets sont additifs et par conséquent qu'il est possible d'isoler et de quantifier les seuls effets de diffusion hydrodynamique induit par cisaillement. Qualitativement nous pouvons conclure que la diffusion hydrodynamique peut être considérée comme l'effet dominant responsable de la migration transverse non spécifique. 


\section{Focalisation hydrodynamique}

\subsection{Principe}

Le transport par diffusion hydrodynamique, et dans une moindre mesure les forces de portance, limitent dans la plupart des cas l'efficacité de la séparation. Le profil des vitesses dans l'épaisseur de la cellule de SPLITT est un profil de Poiseuille. Les particules étant injectées près des parois, elles vont donc subir un cisaillement maximal donc une diffusion hydrodynamique maximale. Nous avons par conséquent mis au point un nouveau séparateur inspiré de la cellule de SPLITT mais où cette fois les particules sont injectées au centre du canal dans une région où le cisaillement est minimal (entrée $\mathrm{c}^{\prime}$ ). Le liquide vecteur est injecté en $\mathrm{a}^{\prime}$ et $\mathrm{b}^{\prime}$. Les particules sont alors focalisées sur l'axe central du canal et s'écoulent sous la forme d'une «nappe » dont l'épaisseur $\delta W_{\mathrm{c}^{\prime}}$ à l'entrée est contrôlée par les débits de focalisation $Q_{\mathrm{a}^{\prime}}$ et $Q_{\mathrm{b}^{\prime}}$. De plus, la nappe présentera un certain élargissement, passant de $\delta W_{\mathrm{c}^{\prime}}$ à $\delta W_{\mathrm{c}}$ au niveau du diviseur de débit à la sortie (Fig. 5).

\subsection{Procédure expérimentale}

L'injection du liquide vecteur, de l'eau ultra pure, aux entrées $a^{\prime}$ et $b^{\prime}$ se fait au moyen de deux pompes à seringues, une pompe péristaltique assurant quant à elle l'injection de l'échantillon en $\mathrm{c}^{\prime}$. Une pompe aspirante est connectée à la sortie a, la sortie b étant laissée libre. La cellule de focalisation possède deux entrées donc deux plans séparateurs (ISP1 et ISP2) qui vont délimiter la nappe de particules à l'entrée du canal (Fig. 5). L'équation reliant le débit d'entrée $Q_{\mathrm{a}^{\prime}}$ (normalisé par le débit total $Q$ ) avec la position $W_{\mathrm{a}^{\prime}} / W$ du plan ISP1 s'obtient en intégrant le champ de vitesse, supposé parabolique :

$$
\frac{Q_{\mathrm{a}^{\prime}}}{Q}=3\left(\frac{W_{\mathrm{a}^{\prime}}}{W}\right)^{2}-2\left(\frac{W_{\mathrm{a}^{\prime}}}{W}\right)^{3}
$$

La solution est alors donnée par :

$$
\frac{W_{\mathrm{a}^{\prime}}}{W}=\sin \left(\frac{\theta}{3}\right)+\frac{1}{2}
$$

avec

$$
\sin \theta=2\left(\frac{Q_{\mathrm{a}^{\prime}}}{Q}\right)-1
$$

La position du plan ISP2 et du plan OSP se calcule de façon identique. On obtient toujours une relation bijective et croissante entre les débits et les positions des plans de séparation d'entrée et de sortie. La focalisation et l'épaisseur de la nappe à l'entrée sont ainsi déterminées par le réglage des débits $Q_{\mathrm{a}^{\prime}}$ et $Q_{\mathrm{b}^{\prime}}$. Le débit $Q_{\mathrm{c}^{\prime}}$ se déduit de la conservation des débits $Q=Q_{\mathrm{a}^{\prime}}+Q_{\mathrm{b}^{\prime}}+Q_{\mathrm{c}^{\prime}}$.

La procédure habituellement utilisée est de fixer $Q_{\mathrm{a}^{\prime}}$ et $Q_{\mathrm{b}^{\prime}}$ (généralement pris égaux pour que la nappe près de l'injection soit focalisée au centre de la cellule) et de faire varier la position $W_{\mathrm{a}}$ de l'OSP en faisant varier $Q_{\mathrm{a}} / Q$.

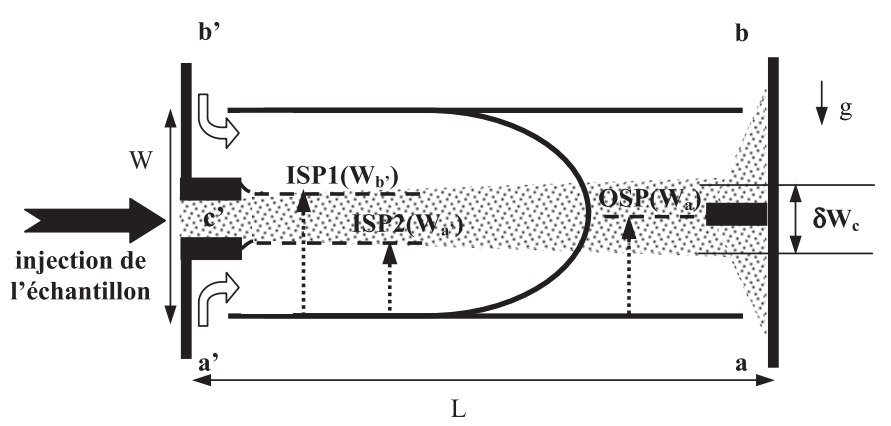

Fig. 5. Vue en coupe de la cellule de focalisation (épaisseur $W=0,731 \mathrm{~mm}$, longueur $L=110 \mathrm{~mm}$, largeur $B=5 \mathrm{~mm}$ ).

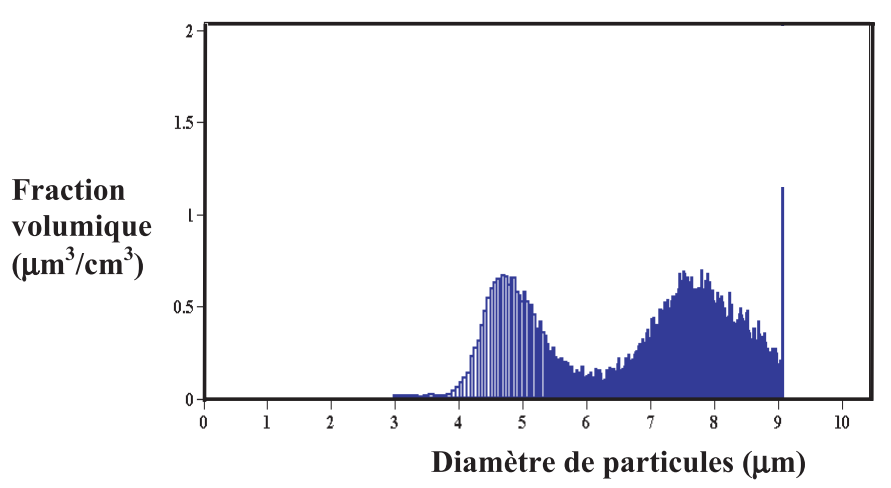

Fig. 6. Histogramme d'un mélange de particules de silice de $5 \mu \mathrm{m}$ et de $8 \mu \mathrm{m}$.

La quantité de particules récoltées aux deux sorties dépendra de la position de l'OSP. Dans l'exemple de la figure 5, l'OSP est placé au centre de la cellule. Nous avons utilisé deux couples de particules : d'une part des particules de silice de $3,5 \mu \mathrm{m}$ et $4,7 \mu \mathrm{m}$ de densité 1,6 g.cm ${ }^{-3}$ et d'autre part des particules de silice de $5 \mu \mathrm{m}$ et $8 \mu \mathrm{m}$ de densité $2,5 \mathrm{~g} \cdot \mathrm{cm}^{-3}$. Le nombre de particules d'une espèce donnée est mesuré à chaque sortie à l'aide d'un compteur de particules et d'analyseur de taille. Un exemple d'histogramme obtenu est représenté sur la figure 6 . Dans ce cas précis, les espèces peuvent être séparées car leur deux fractogrammes en taille sont complètement dissociés.

\subsection{Trajectoires théoriques}

Une séparation binaire parfaite se fera lorsque au niveau du diviseur de débit à la sortie, les deux nappes de particules seront bien séparées. Le réglage des débits de sortie permet ainsi l'élution des fractions séparées par différents ports de sortie comme le montrent les simulations numériques bidimensionnelles de la figure 7. Elles montrent la possibilité de séparer dans un champ gravitationnel deux espèces de particules de tailles différentes mais de même densité dans un profil de vitesses de Poiseuille. Tout en sédimentant, la nappe s'élargit. Pour prendre en compte cet élargissement, nous avons introduit dans les simulations un coefficient de diffusion effectif $D_{\text {eff }}=5 \times 10^{-5} \mathrm{~cm}^{2} / \mathrm{s}$ du même ordre de grandeur que celui déjà obtenu dans une cellule de SPLITT classique [9]. 


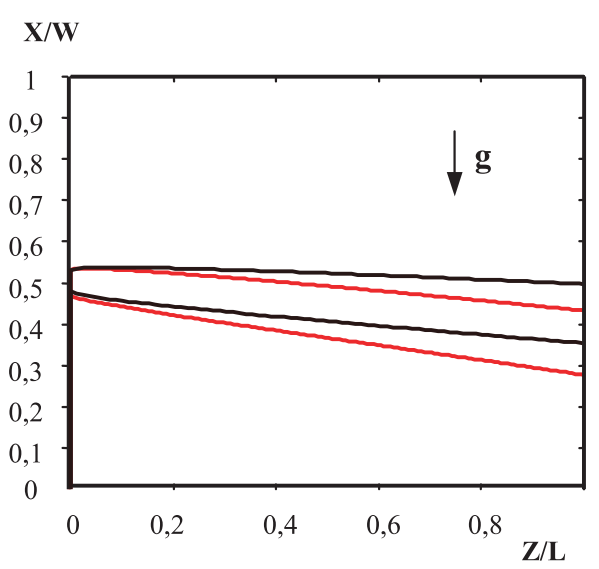

(a)

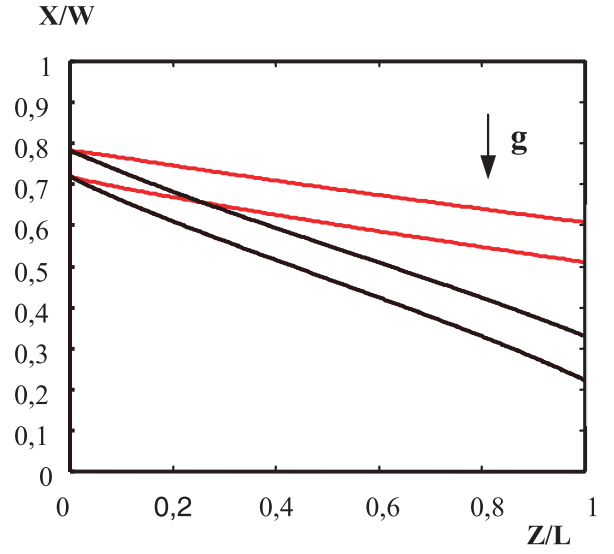

(b)

Fig. 7. Simulation numérique de la trajectoire de deux nappes de particules de différents diamètres, d'épaisseur initiale $80 \mu \mathrm{m}$. L'axe $x$ est suivant l'épaisseur $W$ de la cellule et l'axe $Z$ dans la direction de l'écoulement - (a) $d_{1}=3,5 \mu \mathrm{m}, d_{2}=4,7 \mu \mathrm{m}$, $Q_{\mathrm{a}^{\prime}}=Q_{\mathrm{b}^{\prime}}=0,9 \mathrm{ml} \cdot \mathrm{min}^{-1}, Q_{\mathrm{c}^{\prime}}=0,2 \mathrm{ml} \cdot \mathrm{min}^{-1}$. (b) $d_{1}=5 \mu \mathrm{m}, d_{2}=8 \mu \mathrm{m}, Q_{\mathrm{a}^{\prime}}=1,67 \mathrm{ml} \cdot \mathrm{min}^{-1}, Q_{\mathrm{b}^{\prime}}=1,33 \mathrm{ml} \cdot \mathrm{min}^{-1}$, $Q_{\mathrm{c}^{\prime}}=1 \mathrm{ml} \cdot \mathrm{min}^{-1}$.

La figure 7a montre l'impossibilité, toute théorique, de totalement séparer des particules de $3,5 \mu \mathrm{m}$ et $4,7 \mu \mathrm{m}$ à cause du recouvrement partiel des nappes à la sortie. Par contre, la simulation de la figure $7 \mathrm{~b}$, dans le cas de particules de $5 \mu \mathrm{m}$ et $8 \mu \mathrm{m}$ montre qu'il serait possible d'obtenir une séparation parfaite des deux espèces si nous plaçons l'OSP à une position $W_{\mathrm{a}} / W$ comprise entre 0,33 et 0,51 . Dans ce dernier cas, nous avons réglé les débits d'entrée du liquide vecteur en $\mathrm{a}^{\prime}$ et $\mathrm{b}^{\prime}\left(Q_{\mathrm{a}^{\prime}}>Q_{\mathrm{b}^{\prime}}\right)$ de façon à ce que les nappes de particules se positionnent pratiquement dès l'injection dans le quart supérieure de la cellule en $X / W \approx 0,7$. Si tel n'était pas le cas, $Q_{\mathrm{a}^{\prime}}=Q_{\mathrm{b}^{\prime}}$ par exemple, on observerait une sédimentation des particules de $8 \mu \mathrm{m}$ sur la paroi inférieure avant qu'elles aient atteint l'une des deux sorties. Pour cette position du faisceau, la diffusion induite par cisaillement n'est malheureusement plus négligeable, sans être toutefois trop importante. Il s'agit par conséquent de trouver un compromis entre la taille de la cellule et le débit total afin de déterminer une position optimale des faisceaux à l'entrée, à un endroit où le cisaillement n'est pas encore trop important, et qui permette une bonne séparation des deux faisceaux à la sortie. Avec les dimensions de notre cellule et la limitation du débit des pompes $\left(4 \mathrm{ml} \cdot \mathrm{min}^{-1}\right.$ pour le débit maximum), les trajectoires théoriques pour ces deux types de particules $(5 \mu \mathrm{m}$ et $8 \mu \mathrm{m})$ nous donnent une position optimale de $0,75 \mathrm{~W}$.

Les résultats de ces simulations ne nous renseignent que sur l'éventuelle faisabilité d'une telle séparation binaire, étant bien entendu qu'elles ne prennent pas en compte les interactions des particules entre elles.

\subsection{Résultats expérimentaux}

\subsubsection{Particules de $3,5 \mu \mathrm{m}$ et $4,7 \mu \mathrm{m}$}

Le premier objectif de la séparation d'un mélange binaire (dans notre cas, particules de diamètre $d_{1}$ et $d_{2}$ et de même densité), est l'obtention d'une espèce pure par l'une des deux sorties. Mais avant de séparer le mélange, il faut en premier lieu déterminer les conditions optimales de séparation, c'est-à-dire commencer par injecter séparément chaque espèce de particules. On détermine ainsi la position optimale de l'OSP et donc la valeur optimale du débit à la sortie $Q_{\mathrm{a}}$. Cette valeur optimale consiste à récupérer lors de l'injection des particules $d_{1}$ le maximum de particules en b (ou en a) et lors de l'injection des particules $d_{2}$ le minimum de particules en b (ou a). Ceci afin d'obtenir la plus grande purification possible lorsque l'on injecte le mélange de $d_{x}$ et de $d_{y}$.

Nous avons commencé par injecter séparément des particules de silice, parfaitement sphériques de diamètre $3,5 \mu \mathrm{m}$ et $4,7 \mu \mathrm{m}$, à $0,5 \%$ en fraction volumique. Les fractions massiques $F_{\mathrm{a}}$ (égale à la masse de particules d'une espèce donnée $d_{\mathrm{i}}$ sortant en a sur la masse totale de particules $d_{\mathrm{i}}$ injectées) en fonction du débit normalisé $Q_{\mathrm{a}} / Q$ sont représentées sur la figure 8. On constate que les deux courbes sont pratiquement confondues aux incertitudes expérimentales près, les tailles des particules étant beaucoup trop proches pour pouvoir espérer les séparer en injectant le mélange. Ceci est cohérent avec les simulations de trajectoires de la figure $7 \mathrm{a}$. Il est vrai qu'en modifiant la valeur de certains paramètres, comme en diminuant le débit total ou en augmentant la longueur de la cellule, on pourrait obtenir deux nappes de particules dissociées à la sortie. Ceci est d'autant crucial que l'injection se situant au centre de la cellule, donc dans la zone de vitesse maximale, le temps de séjour des particules est plus faible que dans le cas d'une injection près des parois. Diminuer le débit pose des problèmes expérimentaux, comme le piégeage des particules dans les différents tuyaux d'entrée et de sortie ou au niveau du séparateur de sortie de la cellule. La construction d'une nouvelle cellule plus longue est donc en cours afin de pouvoir séparer des particules de taille très proche. 


\section{$\mathbf{F}_{\mathbf{a}}$}

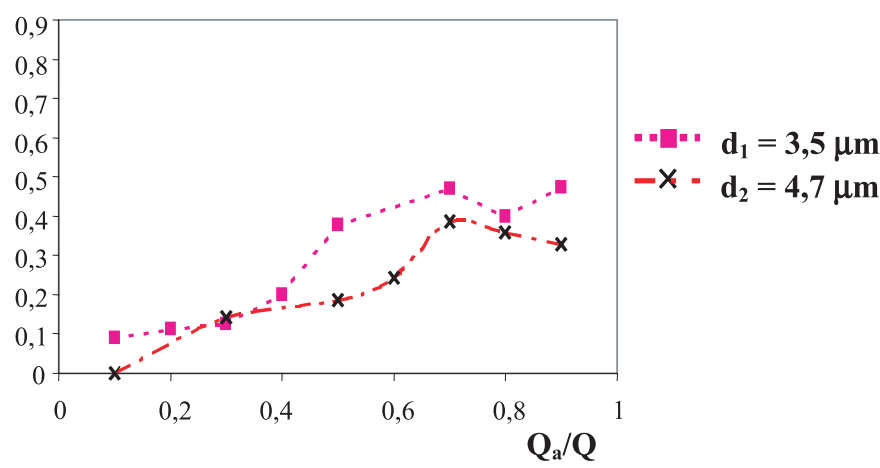

Fig. 8. Fraction massique $F_{\text {a }}$ de particules de silice de densité $1,6 \mathrm{~g} \cdot \mathrm{cm}^{-3}$ en fonction du débit normalisé à la sortie a.

\subsubsection{Particules de silice de $5 \mu \mathrm{m}$ et $8 \mu \mathrm{m}$}

\section{Comportement individuel}

L'augmentation de la différence de taille entre les particules permet d'éviter le recouvrement des deux nappes à la sortie du séparateur, comme dans le cas de la figure $7 \mathrm{~b}$. Nous avons donc injecté séparément une première solution de particules de silice de $5 \mu \mathrm{m}$ puis une deuxième solution de $8 \mu \mathrm{m}$ de même densité $\left(2,5 \mathrm{~g} . \mathrm{cm}^{-3}\right)$ dans les mêmes conditions $\left(Q_{\mathrm{a}^{\prime}}=1,67 \mathrm{ml} \cdot \mathrm{min}^{-1}, Q_{\mathrm{b}^{\prime}}=1,33 \mathrm{ml} \cdot \mathrm{min}^{-1}\right.$ et $\left.Q_{\mathrm{c}^{\prime}}=1 \mathrm{ml} \cdot \mathrm{min}^{-1}\right)$. La figure 9 représente les fractions massiques $F_{\mathrm{a}}$ et $F_{\mathrm{b}}$ en fonction de la position de l'OSP normalisée $W_{\mathrm{a}} / W$. Le débit de sortie en a étant contrôlé par une pompe aspirante, le nombre de particules sortant en a augmente (tandis que celui sortant en b diminue) lorsque l'aspiration $Q_{\mathrm{a}}$ augmente ou quand la position $W_{\mathrm{a}} / W$ de l'OSP augmente. Dans le cas des particules de $8 \mu \mathrm{m}$, presque tout l'échantillon injecté est récupéré en a, environ $90 \%$, pour une position optimale de l'OSP comprise entre $0,7 \mathrm{~W}$ et $0,8 \mathrm{~W}$. Le comportement des particules de $5 \mu \mathrm{m}$ est différent : à partir de $W_{\mathrm{a}} / W=0,5$, le même nombre sensiblement de particules sortent en a et en b ce qui signifie que la moitié de l'échantillon n'a pas eu le temps de sédimenter pour atteindre la sortie inférieure a. Ce résultat est qualitativement en accord avec la simulation de la figure $7 \mathrm{~b}$.

\section{Comportement collectif (injection du mélange)}

La séparation idéale n'est pas forcément d'obtenir une espèce pure à chaque sortie (toutes les particules de $8 \mu \mathrm{m}$ en a et toutes les particules de $5 \mu \mathrm{m}$ en b par exemple) mais à au moins une des sorties. Afin de quantifier la pureté de chaque espèce collectée à chaque sortie, on définit le facteur d'enrichissement $e$ (ou d'appauvrissement) des particules de taille $d_{\mathrm{i}}$ à la sortie a (ou b) comme :

$$
e\left(d_{\mathrm{i}}\right)=\frac{\text { Nombre de particules } d_{\mathrm{i}} \text { à la sortie a }(\mathrm{ou} \mathrm{b})}{\text { Nombre total de particules à la sortie a }(\mathrm{ou} \mathrm{b})}
$$

Si ce facteur $e$ est supérieur à $1 / 2$, on aura un enrichissement de l'espèce considérée à la sortie considérée, dans le cas contraire un appauvrissement. La purification de l'espèce $d_{\mathrm{i}}$ sera parfaite lorsque $e=1$.

Le mélange a été préparé avec un nombre identique de particules de silice de $5 \mu \mathrm{m}$ et de $8 \mu \mathrm{m}$. Il est injecté au centre de la cellule de focalisation avec un débit $Q_{\mathrm{c}^{\prime}}=$ $1 \mathrm{ml} \cdot \mathrm{min}^{-1}\left(Q_{\mathrm{a}}^{\prime}=1,67 \mathrm{ml} \cdot \mathrm{min}^{-1}, Q_{\mathrm{b}}^{\prime}=1,33 \mathrm{ml} \cdot \mathrm{min}^{-1}\right)$. Le résultat est représenté sur la figure 10a. Pour une position de l'OSP d'environ 0,8 W, $40 \%$ des particules de $5 \mu \mathrm{m}$ et $90 \%$ environ des particules de $8 \mu \mathrm{m}$ sont collectées à la sortie inférieure a, tandis que $60 \%$ des particules de $5 \mu \mathrm{m}$ et $10 \%$ des particules de $8 \mu \mathrm{m}$ sont éluées par la sortie b. On se retrouve ainsi avec un enrichissement des particules de $5 \mu \mathrm{m}$ en b : $e(d=5 \mu \mathrm{m})=0,86$.

Malgré tout, une pureté parfaite n'est pas encore atteinte. Pour augmenter ce facteur $e$, il faudrait réinjecter le mélange de particules obtenu, puis répéter l'opération autant de fois qu'il est nécessaire jusqu'à l'obtention d'une pureté quasi parfaite. Une autre façon de faire qui nécessiterait moins de manipulations, serait de connecter entre elles plusieurs petites cellules de focalisation, dont le nombre dépendrait du degré de purification à chaque sortie.

\subsubsection{Efficacité comparée de la séparation par SPLITT et par focalisation}

Notre principale motivation dans la mise au point de la cellule de focalisation était d'une part de tester la faisabilité d'une séparation binaire, et d'autre part de comparer son efficacité avec une séparation dans une cellule de SPLITT classique. Afin de respecter la même procédure expérimentale, nous avons injecté dans la cellule de SPLITT placée horizontalement le même mélange que dans la cellule de focalisation (même proportion de particules de $5 \mu \mathrm{m}$ et $8 \mu \mathrm{m}$ ) avec un débit total de $4 \mathrm{ml} \cdot \mathrm{min}^{-1}$. Les résultats sont reportés sur la figure $10 \mathrm{~b}$. On constate que pour une position optimale de l'OSP, $W_{\mathrm{a}} / W=0,8$, le pourcentage de particules récupérées à la sortie supérieure b est de $30 \%$ et de $50 \%$ pour les particules de $8 \mu \mathrm{m}$ et de $5 \mu \mathrm{m}$, ce qui conduit à un facteur d'enrichissement $e(d=5 \mu \mathrm{m})=0,625$. Cette valeur, comparée à celle obtenue en focalisation $(e=0,86)$ est nettement plus faible : dans les mêmes conditions opératoires, l'efficacité d'une séparation binaire par la technique de focalisation est donc augmentée de $37 \%$.

\section{Conclusion}

Nous avons montré, notamment par des expériences réalisées en micropesanteur le rôle essentiel joué par la diffusion hydrodynamique induite par cisaillement dans la migration non spécifique. Nous avons ainsi mis au point une nouvelle méthode de séparation sans membrane par focalisation hydrodynamique. Celle-ci présente plusieurs avantages par rapport à une cellule de SPLITT classique : après injection des particules au centre du canal, il est possible de les focaliser rapidement dans n'importe quelle 


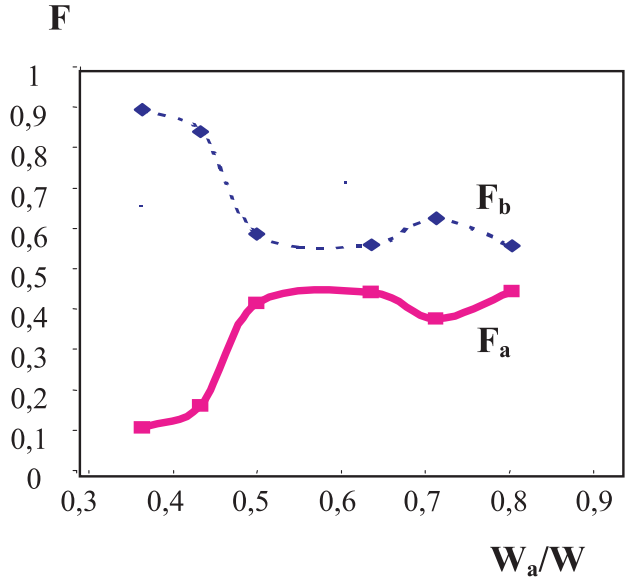

(a)

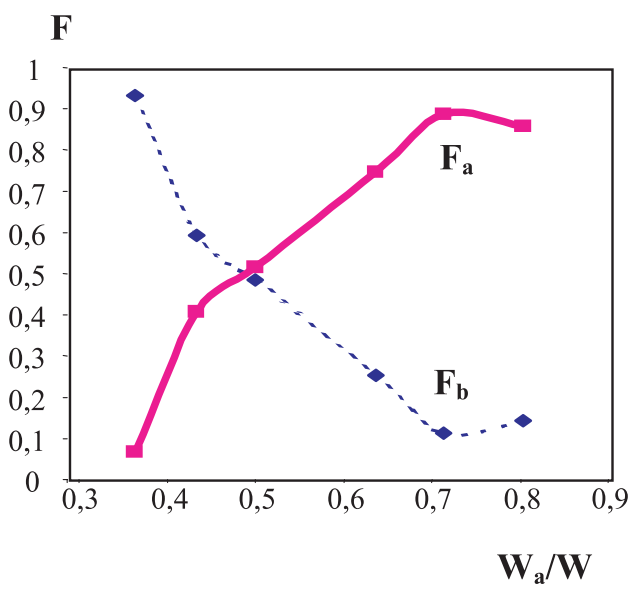

(b)

Fig. 9. Fractions massiques récupérées aux sorties a et b en fonction de la position de l'OSP : (a) Particules de silice de $5 \mu$ m - (b) Particules de silice de $8 \mu \mathrm{m}$.

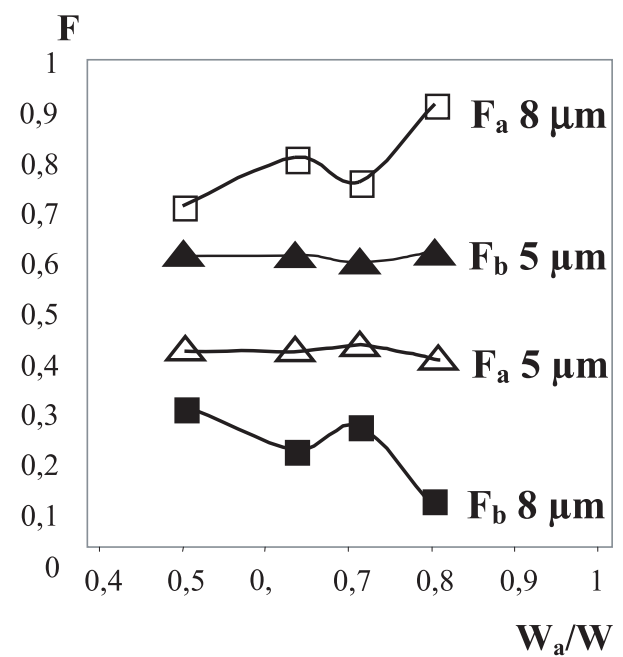

(a)

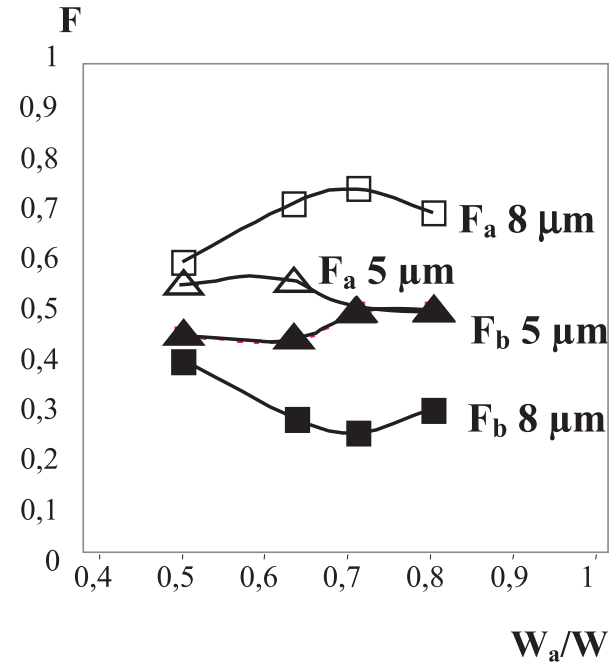

(b)

Fig. 10. Comparaison des fractions massiques récupérées aux sorties a et b pour un mélange de particules de silice de $5 \mu \mathrm{m}$ et $8 \mu \mathrm{m}$ - (a) Expérience de focalisation - (b) Expérience de SPLITT.

position dans l'épaisseur de la cellule simplement en ajustant les deux débits d'entrée du liquide vecteur. En particulier, la position du faisceau est optimisée de façon à avoir une diffusion (induite par cisaillement) la plus faible possible tout en tenant compte des contraintes liées à l'expérience.

Les premiers résultats de séparation binaire, malgré un faisceau focalisé à 0,75 fois l'épaisseur de la cellule, ont montré une forte augmentation de l'efficacité par rapport à celle obtenue par la technique de SPLITT (environ $36 \%$ ), ouvrant ainsi la voie à de nombreuses applications, notamment biomédicales (séparation plaquettaire, séparation des cellules souches du sang, isolement des cellules cancéreuses).

Remerciements. Les auteurs tiennent à remercier le CNES pour l'aide financière apportée à la réalisation de cette étude.

\section{Références}

[1] C. Giddings, Optimization of transport_driven continuous SPLITT fractionation, Sep. Sci. Technol. 27(11) (1992) 1489-1504

[2] M. Hoyos, K. Mc Closkey, M. Moore, M. Nakamura, B. Bowell, J. Chalmers, M. Zborowski, Pulse injection studies of blood progenitor cells in quadrupole magnetic flow sorter, Sep. Sci. Technol. 37(4) (2002) 745-767

[3] M. Hoyos, P. Kurowski, D. Salhi, L. Moore, S. Mills, M. Zborowski, Characterization of the non-specific crossover in SPLITT fractionation, soumis à J. Chromatography A, 2003

[4] S. Williams, L. Moore, J. Chalmers, M. Zborowski, Splitter Imperfection un annular split-flow channel separation cells: effect on non-specific crossover, soumis à Anal. Chem. (2003) 
[5] P. Ho, G. Leal, Inertial migration of rigid spheres in twodimensional unidirectional flows, J. Fluid Mech. 65 (1974) 365-400

[6] P. Vasseur, G. Cox, The lateral migration of a spherical particle in two dimensional shear Flow, J. Fluid Mech. 78 (1976) 385-413

[7] G. Segré, A. Silberberg, Behaviour of macroscopic rigid spheres in Poiseuille flow, Parts 1 and 2, J. Fluid Mech. 14 (1962) 115-136

[8] U. Zhang, S. Williams, M. Myers, C. Giddings, Separation of cells and cell-sized particles by continuous SPLITT fractionation using hydrodynamic lift forces, Sep. Sci. Technol. 29(18) (1994) 2493-2522

[9] M. Hoyos, P. Kurowski, Rôle des forces de portance et diffusion hydrodynamique dans les processus de séparation par la technique de SPLITT, J. Phys. IV France 11 (2001) Pr6-73-Pr6-81

[10] D Leighton, A. Acrivos, Viscous resuspension, Chem. Eng. Sci. 41(6) (1986) 1377-1384

[11] E. Eckstein, D. Bailey, A. Shapiro, Self-diffusion of particles in shear flow of a suspension, J. Fluid Mech. 79 (1977) 191-208

[12] D. Leighton, A. Acrivos, The shear-induced migration of particles in concentrated suspensions, J. Fluid Mech. 181 (1987) 415-439

[13] D. Leighton, A. Acrivos, Measurement of shear-induced self-diffusion in concentrated suspensions of spheres, J. Fluid Mech. 177 (1987) 109-131 